\title{
Dynamically Controlled Resonance Fluorescence Spectra from a Doubly Dressed Single InGaAs Quantum Dot
}

\author{
Y. He ${ }^{1,2}$ Y.-M. He, ${ }^{1,2}$ J. Liu,,${ }^{3,4,}$ Y.-J. Wei, ${ }^{1,2}$ H. Y. Ramírez, ${ }^{1,2}$ M. Atatüre,${ }^{5,1}$ C. Schneider, ${ }^{6}$ M. Kamp, ${ }^{6}$ \\ S. Höfling, ${ }^{6,1,2,7}$ C.-Y. Lu, ${ }^{1,2, \dagger}$ and J.-W. Pan ${ }^{1,2, \$}$ \\ ${ }^{1}$ Hefei National Laboratory for Physical Sciences at Microscale and Department of Modern Physics, \\ University of Science and Technology of China, Hefei, Anhui 230026, China \\ ${ }^{2}$ CAS Centre for Excellence and Synergetic Innovation Centre in Quantum Information and Quantum Physics, \\ University of Science and Technology of China, Hefei, Anhui 230026, China \\ ${ }^{3}$ Institute for Research in Electronics and Applied Physics, University of Maryland, College Park, Maryland 20742, USA \\ ${ }^{4}$ South China Academy of Advanced Optoelectronics, South China Normal University, Guangdong, Guangzhou 510006, China \\ ${ }^{5}$ Cavendish Laboratory, JJ Thomson Avenue, University of Cambridge, CB3 OHE Cambridge, United Kingdom \\ ${ }^{6}$ Technische Physik, Physikalisches Instität and Wilhelm Conrad Röntgen-Center for Complex Material Systems, \\ Universitat Würzburg, Am Hubland, D-97074 Wüzburg, Germany \\ ${ }^{7}$ SUPA, School of Physics and Astronomy, University of St. Andrews, St. Andrews KY16 9SS, United Kingdom
}

(Received 15 November 2014; published 4 March 2015)

\begin{abstract}
We report the first experimental demonstration of the interference-induced spectral line elimination predicted by Zhu and Scully [Phys. Rev. Lett. 76, 388 (1996)] and Ficek and Rudolph [Phys. Rev. A 60, R4245 (1999)]. We drive an exciton transition of a self-assembled quantum dot in order to realize a twolevel system exposed to a bichromatic laser field and observe the nearly complete elimination of the resonance fluorescence spectral line at the driving laser frequency. This is caused by quantum interference between coupled transitions among the doubly dressed excitonic states, without population trapping. We also demonstrate a multiphoton ac Stark effect with shifted subharmonic resonances and dynamical modifications of resonance fluorescence spectra by using double dressing.
\end{abstract}

Self-assembled semiconductor quantum dots (QDs) have attracted considerable interest in both fundamental studies of quantum optics and promising applications in optoelectronics and quantum information [1-3], as their atomlike properties can be tailored artificially through epitaxial growth and band-gap engineering. Their compatibility with semiconductor nanofabrication technologies enables monolithical integration to micro- and nanostructures to enhance light-matter interaction [4]. The atomlike behaviors of QDs have been confirmed by numerous experiments such as photon antibunching [5,6] and spontaneous emission control $[7,8]$. More recently, coherent control of the QD two-level systems has allowed the observations of Rabi oscillation [9], Ramsey interference [10], AutlerTownes splitting [11], Mollow absorption [12], and a resonance fluorescence triplet [13-15], which can be well preserved even under strong optical excitations. Resonance fluorescence from QDs has also provided a versatile tool in spectroscopy [16,17] and spin readout [18], and as a high-quality single-photon source [19-21].

New physics beyond the Mollow fluorescence triplet arises when a two-level system is exposed to strong bichromatic driving fields, exhibiting a range of nonlinear and multiphoton dynamics of light-matter interaction $[22,23]$. In particular, it has been pointed out that a dynamical cancellation of the spontaneous emission spectral line, initially predicted for a three-level system [24,25], may be possible in a two-level atom [26,27], which is not due to population trapping, but induced by quantum interferences between different transition channels in a doubly dressed atom. This occurs when a two-level atom is driven by a strong resonant laser with a Rabi frequency $2 \Omega$ [28], and a second, weaker laser is detuned by $2 \Omega$ from the strong field. The suppression of spontaneous emission spectral lines also appears when the second laser is coupled to the shifted subharmonics of $2 \Omega$, which can be attributed to the multiphoton ac Stark effect [22]. Despite the extensive efforts undertaken to observe this phenomenon [29-34], the experimental demonstration of a complete cancellation of spontaneous emission lines has been claimed once in a $V$-type level atom [29], where there was also an alternative interpretation based on population trapping possible [32]. Here, we use a single QD, doubly dressed by bichromatic fields, to demonstrate unequivocally the complete cancellation of spontaneous emission spectral lines and other features arising from this phenomenon including shifted subharmonic resonances and modifications of sideband spectra.

Our experiments are carried out on self-assembled InGaAs QDs embeded in a low- $Q$ planar microcavity $(Q \approx 200)$ and housed in a cryogen-free bath cryostat operating at $4.2 \mathrm{~K}$, as shown in Fig. 1(a). The measurements are performed on the neutral exciton transition of a single QD with a confocal microscope allowing bichromatic excitations of the QD from the side arm and collection of 
(a)

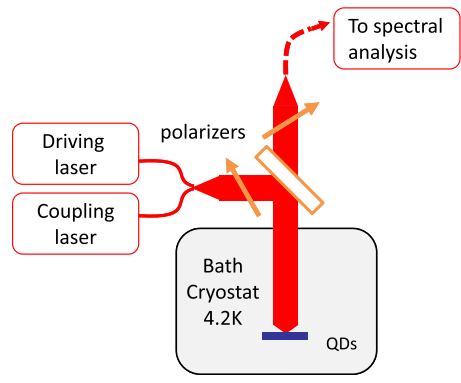

(c)

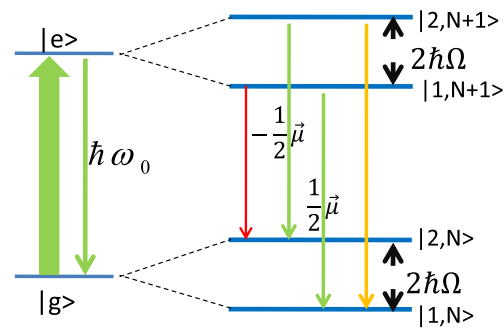

(b)

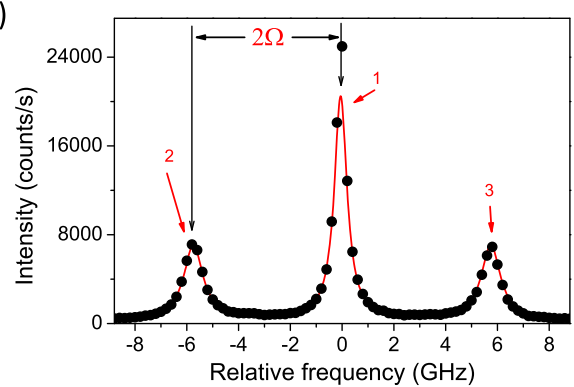

(d)

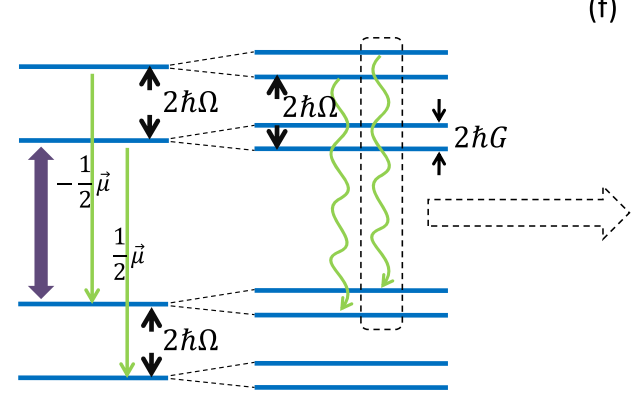

(e)

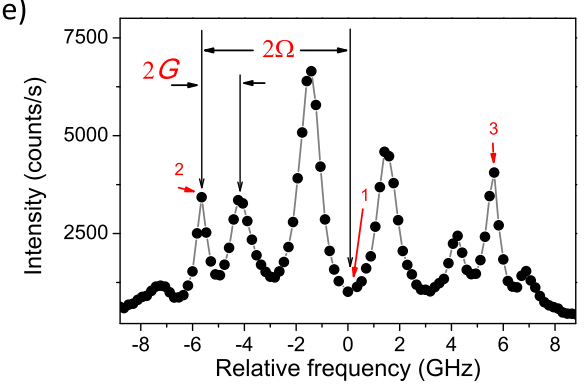

(f)

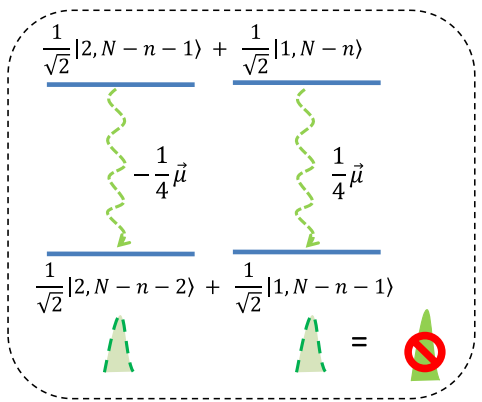

FIG. 1 (color online). Resonance fluorescence: from Mollow triplet to the doubly dressed spectrum. (a) Schematic of the experiment setup. (b) Dressed QD spectrum showing a typical Mollow triplet. The splitting is the Rabi frequency of the driving laser $2 \Omega=5.8 \pm 0.1 \mathrm{GHz}$. Black circles are the experimental data and the red curve is the theoretical fit. (c) Energy levels of singly dressed states of the QD excited by a strong resonant pumping laser (thick green arrow). The ground state $|g\rangle$ and excited state $|e\rangle$ of the QD are split into doublet states with a splitting energy $2 \hbar \Omega$. The two transitions related to the central peak of the Mollow triplet are antiparallel (green lines), but decay with no correlation. The yellow and red lines correspond to two side-peak transitions. (d) Energy levels of the doubly dressed states of the QD by introducing a coupling laser (thick purple arrow) resonant to the inner doublet transition. Every singly dressed state splits into new doublets with a spacing of $2 \hbar G$, where $2 G$ is the coupling field Rabi frequency. The transitions corresponding to the central peak in the doubly dressed spectrum are represented in green wavy arrows. (e) Doubly dressed QD spectrum. The central peak is strongly suppressed. The splitting of the peaks within the daughter triplet is $2 G=0.6 \Omega$. (f) Tracing the transition, from $|N+M, n-\rangle$ to $|N+M-1, n-\rangle$, back to their eigenstates. The selection-rules-allowed transitions are indicated by dashed wavy arrows. These two transitions possess opposite phases and destructively interfere, which leads to the suppression of the spontaneous emission at the driving laser frequency (for simplicity, the weak field photon number $M$ is abbreviated and not shown).

the emitted fluorescence through the top arm. By using a cross-polarization configuration the resonance fluorescence is separated from the excitation lasers with a signal to background ratio exceeding 28:1 for the highest excitation power of $100 \mu \mathrm{W}\left(2 \Omega \approx 14.2 \gamma_{\mathrm{sp}}\right)$ used in this experiment, in which $\gamma_{\mathrm{sp}}$ denotes the spontaneous emission rate of the neutral exciton state.

Figure 1(b) shows a typical Mollow triplet spectrum from a single QD resonantly driven by a strong, narrowband $(<2 \mathrm{MHz})$ laser. Exciton states in QDs are treated as two-level systems with ground state $|g\rangle$, excited state $|e\rangle$, transition frequency $\omega_{0}$, and dipole transition moment $\vec{\mu}$. Because of the light-matter interaction induced by the pumping laser, the dressed states (doublets) are formed:

$$
|i, N\rangle=\frac{1}{\sqrt{2}}\left[|g, N\rangle-(-1)^{i}|e, N-1\rangle\right], \quad i=1,2
$$

with energies $E_{i, N}=\hbar\left(N \omega_{0} \pm \Omega\right)$, where $N$ is the number of photons in the driving field and $2 \Omega$ is the Rabi frequency. The four transitions between neighboring doublets result in three distinct transition frequencies $\omega_{0}$ and $\omega_{0} \pm 2 \Omega$ in the
Mollow triplet spectrum, see Fig. 1(c). The exciton lifetime $T_{1}=390 \mathrm{ps}$ is obtained from time-resolved measurements while the Rabi frequency of the driving laser $2 \Omega=5.8 \pm$ $0.1 \mathrm{GHz}$ and the coherence time of the exciton state $T_{2}=$ 424 ps are extracted by fitting the Mollow triplet spectrum (see Sec. 3 of the Supplemental Material [35]). It is worthwhile to notice that the two transitions contributing to the central peak exhibit antiparallel dipole moments, i.e., $\pm \frac{1}{2} \vec{\mu}$, but the calculated correlation functions of these transitions $\left\langle\sigma_{i i N}^{+} \sigma_{j j N}^{-}\right\rangle(i \neq j)$ are equal to zero [26], indicating these two transitions decay independently. $\sigma_{i i N}^{ \pm}=$ $|i, N\rangle\langle N-1, j|(i, j=1,2)$ is the transition dipole moment operator of the dressed atom transition.

We then introduce a second coupling laser with frequency $\omega_{2}=\omega_{0}-2 \Omega$ and Rabi frequency $2 G=0.6 \Omega$ to couple the degenerate transitions that relate to the central peak, as shown in Fig. 1(d). The newly formed doubly dressed states can be written as $|N+M, n \pm\rangle=$ $(1 / \sqrt{2})[|2, N-n-1, M+n+1\rangle \pm|1, N-n, M+n\rangle]$, where $N, M$ are the photon numbers of the driving field and the coupling field, respectively, and $n$ is the sublevel number within the doublets. Every spectral emission line 
in the Mollow triplet splits into a daughter triplet because of the formation of quartet states by dressing the doublet states, as shown in Fig. 1(d). In such a spectrum nine emission lines in total are expected; however, in the measured spectrum shown in Fig. 1(e) the central emission line is completely suppressed as a result of the destructive quantum interference induced by the coupling laser.

This quantum interference is easier to interpret by tracing the transitions between doubly dressed states back to their eigenstates. One of the transitions corresponding to the central peak, from $|N+M, n+\rangle$ to $|N+M-1, n+\rangle$, is highlighted in Fig. 1(d) and traced back to the superimpositions of their eigenstates (singly dressed states) in Fig. 1(f): $|N+M, n+\rangle=(1 / \sqrt{2})[\mid 2, N-n-1, M+n+$ $1\rangle+|1, N-n, M+n\rangle],|N+M-1, n+\rangle=(1 / \sqrt{2})[\mid 2, N-$ $n-2, M+n\rangle+|1, N-n-1, M+n-1\rangle]$. Among the possible decay channels between these eigenstates, only the transitions from $|2, N-n-1, M+n+1\rangle$ to $\mid 2, N-n-$ $2, M+n\rangle$ and from $|1, N-n, M+n\rangle$ to $\mid 1, N-n-$ $1, M+n\rangle$ are allowed by selection rules. Detailed calculations (see Sec. 5 of the Supplemental Material [35]) reveal that these two transitions should be antiphased and interfere destructively, eliminating the central spectral line. Similarly, other degenerate transitions corresponding to the central peak, e.g., the transition from $|N+M, n-\rangle$ to $|N+M-1, n-\rangle$, also exhibit destructive interferences.

A quantitative comparison between the predicted and the measured spectra is performed by scanning the coupling laser across the resonance dip $\left(\omega_{0}-2 \Omega\right)$. Frequency detuning between the coupling laser and inner transition of the doublets is denoted as $\Delta_{2}=\omega_{2}-\left(\omega_{0}-2 \Omega\right)$. Figure 2(a) represents a $2 \mathrm{D}$ contour plot of the $\Delta_{2}$ dependent doubly dressed spectra, which are well reproduced in the simulation of Fig. 2(b) by using the parameters extracted from the experiments (see Sec. 6A of the Supplemental Material [35]). The driving laser detuning $\Delta_{1}=-0.977 \mathrm{GHz}$ used in the simulation is obtained from a fit of the central peak intensity as a function of $\Delta_{2}$ in Fig. 2(a), as shown in Fig. 2(c) (see Sec. 6B of the

(a)

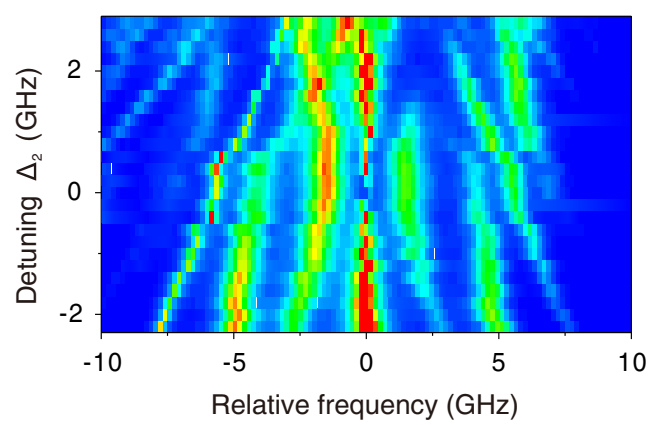

(b)

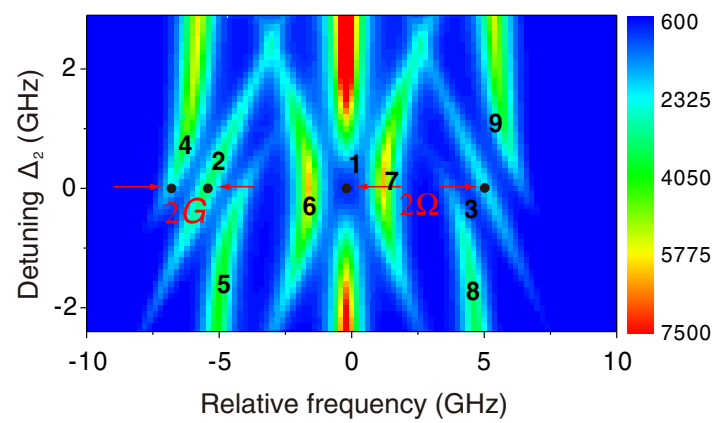

Supplemental Material [35]). Comparing the experimental spectra to the prediction, we find that both the peak evolution trends and their corresponding strengths agree remarkably well. The anticrossing feature of peaks 4,5 (6,7 or 8,9) in Fig. 2(b) is an indication of the coupling laser induced interaction while peaks 2,3 evolve linearly with coupling laser detuning $\Delta_{2}$. As the coupling laser moves away from the resonance, the center peak suppressed by quantum interference gradually emerges and the Mollow triplet recovers at large detuning ranges.

To investigate the multiphoton absorption process and spectral eliminations at subharmonic resonances, we tune the coupling laser from the bare exciton energy $\omega_{0}$ to the inner doublet transition $\omega_{0}-2 \Omega$; i.e., the frequency difference between the coupling laser and the bare exciton energy $\Delta_{3}=\omega_{2}-\omega_{0}$ is tuned from zero to $-2 \Omega$. A $140-\mathrm{MHz}$ etalon with a $9.18 \mathrm{GHz}$ free spectral range is employed to filter out the central peak fluorescence signal when tuning the coupling laser. The center peak intensity as a function of $-\Delta_{3}$ is plotted in Fig. 3 and spectral line suppressions are clearly observed near to the subharmonic Rabi frequencies $2 \Omega / n(n=1-5)$. Instead of quantum interference induced by single photons, here the coupling laser via an $n$-photon process [36] couples the dressed states created by the strong driving field, which results in a dynamic Stark shift of the states and effectively shifts the positions of the subharmonics. The shifts of the subharmonics can be calculated in the framework of high-order perturbation theory by taking all other doublet states within the same energy manifold into account [22] as $\Delta_{1}=(1 / 8) \alpha^{2} \Omega$ for $n=1$ and $\Delta_{n}=$ $n \alpha^{2} \Omega /\left[2\left(n^{2}-1\right)\right]$ for $n>1$, where $\alpha$ is the power ratio between the coupling and driving laser. The calculated frequency displacements are $0.13,0.34$, and $0.19 \mathrm{GHz}$ for $2 \Omega, 2 \Omega / 2$, and $2 \Omega / 3$, respectively, and are very close to the shifts $0.11,0.24$, and $0.20 \mathrm{GHz}$ observed in the experiments. The incomplete suppression of the center peak at subharmonics is an effect of the reduced splitting of the doubly dressed states in higher multiphoton processes [22]. The spectral cancellation induced by quantum interferences is

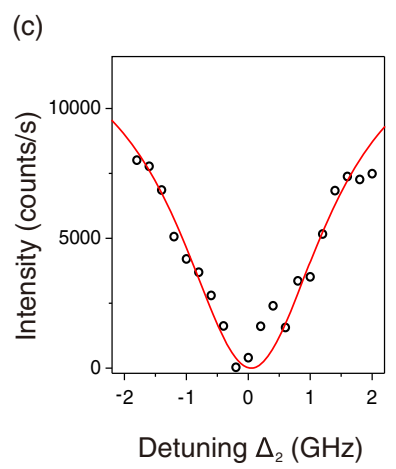

FIG. 2 (color online). Spectra of the doubly dressed QD. (a) Measured spectra of the doubly dressed QD as a function of detuning $\Delta_{2}$. (b) The simulation of doubly dressed spectra. The driving laser Rabi frequency $2 \Omega$ associates with the splitting of the original Mollow triplet peaks 1,2 and 1,3 while the coupling laser Rabi frequency $2 G$ relates to the inner splitting of the peaks in the daughter triplet. (a) and (b) share the same color bar. (c) The central peak intensity versus the coupling laser detuning. Counts at zero detuning go to dark counts level. Black circles are the experimental data and the red curve is the theoretical fit. 


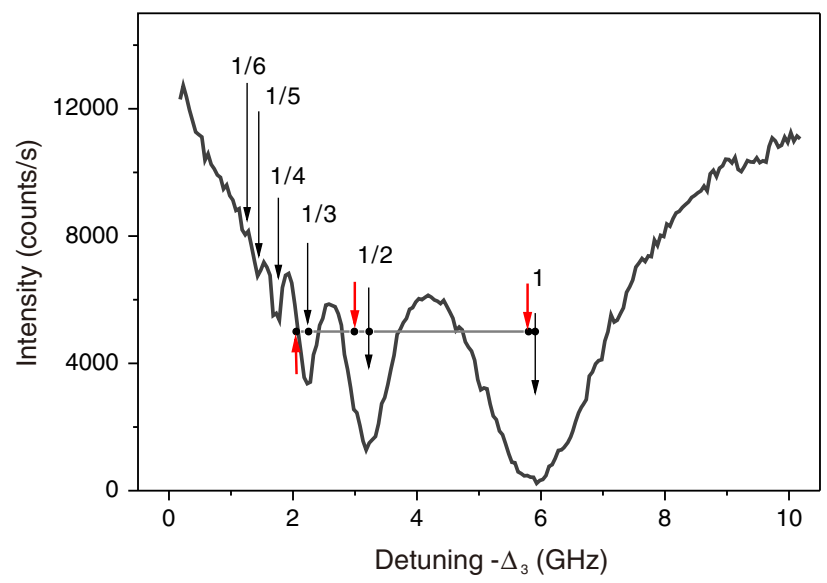

FIG. 3 (color online). Subharmonics of the central peak for the doubly dressed QD. The positions of the subharmonics observed in experiments are indicated by black arrows while the unshifted frequencies for the first three subharmonics are shown by red arrows, exhibiting observable shifts as a result of a multiphoton ac Stark shift.

different from the processes with the population trapped in a specific state, such as coherent population trapping [37] or electromagnetically induced transparency [38]. In our system, population $P_{n \pm, \bar{N}}=\langle \pm n, \bar{N}|\rho| \bar{N}, n \pm\rangle$ is evenly distributed within these states [26], where $|\bar{N}, n \pm\rangle$ is the doubly dressed eigenstate, $\bar{N}=M+N$ is the total photon number, and $\rho$ is the state density matrix.

Finally, the coupling laser is tuned to zero detuning with the exciton energy, i.e., exactly the same frequency as the driving laser. This degenerate excitation scheme generates a modified Mollow triplet in which the two side peaks evolve into flat and broad sidebands, as predicted by Freedhoff and Ficek [39]. Figures 4(a) and 4(c) represent the experimental spectra for the power ratios of $\alpha=0.2$ and $\alpha=0.4$,

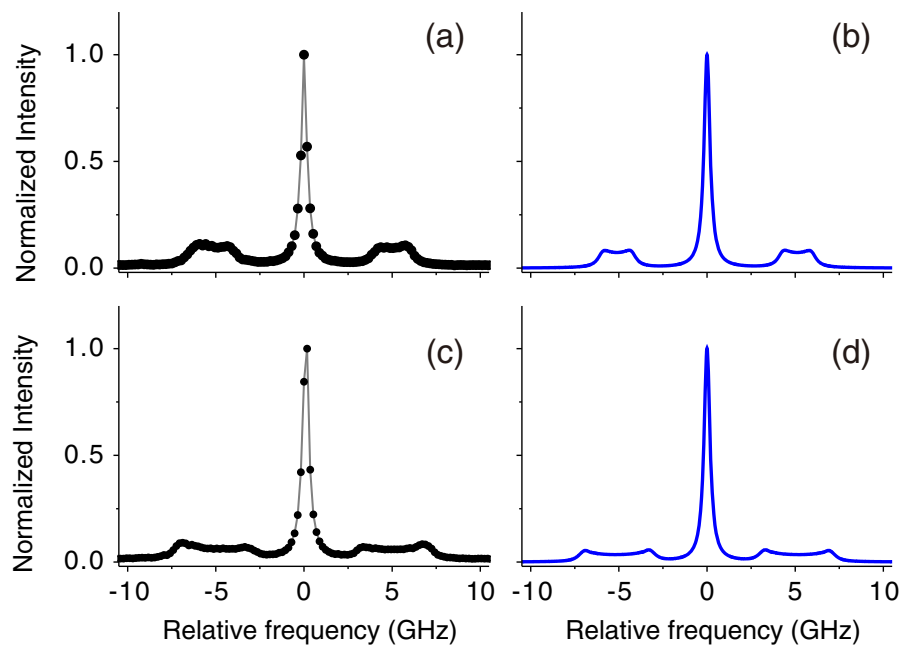

(b)
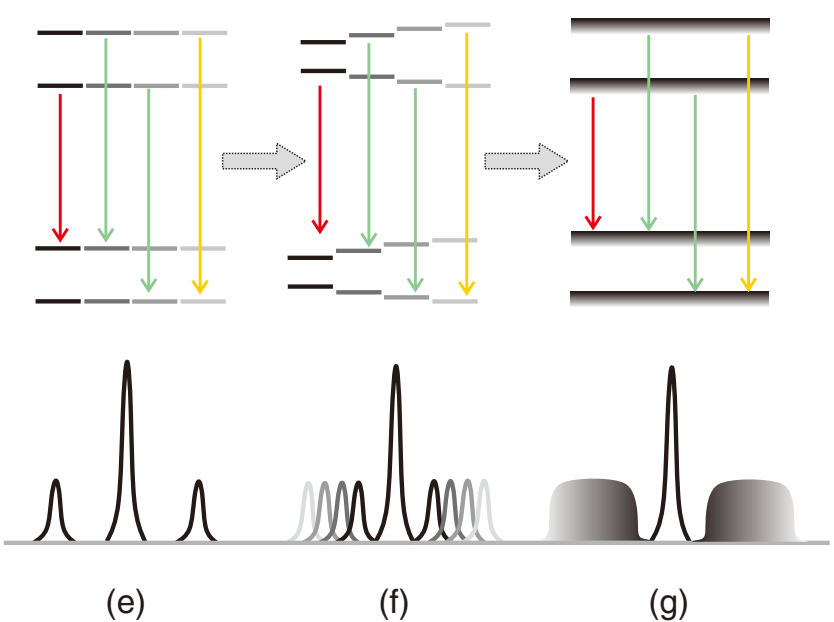

FIG. 4 (color online). Sideband modulation by doubly dressing the QD. (a) and (b) are the experimental and simulated spectra for $\alpha=0.2$, respectively. (c) and (d) are the experimental and simulated spectra for $\alpha=0.4$, respectively. The $x$ axis and $y$ axis are the same in (a)-(d). (e) Energy levels of the doubly dressed QDs without considering the interaction with the coupling laser. (f) Degeneracy lifting of the doubly dressed states by taking the coupling laser into account. (g) Continuum energy levels and flat sidebands of the QD driven by two lasers with the same frequency. 
line narrowing [40], lasing without inversion [41], and quantum entanglement generation [42] can be envisioned in QDs. Along this direction, our work may facilitate the study of complicated spectra of two-level systems under multicolor laser excitations and stimulate research on novel quantum interference phenomena in solid-state quantum systems.

We acknowledge $\mathrm{P}$. Wu, and $\mathrm{M}$. Cramer for useful discussions. This work was supported by the National Natural Science Foundation of China, the Chinese Academy of Sciences, and the National Fundamental Research Program.

Y.H. and Y.-M. H. contributed equally to the work.

*in.liu@ coer-scnu.org

cylu@ustc.edu.cn \#pan@ustc.edu.cn

[1] A. N. Vamivakas and M. Atature, Contemp. Phys. 51, 17 (2010).

[2] S. Buckley, K. Rivoirem, and J. Vuĉković, Rep. Prog. Phys. 75, 126503 (2012).

[3] R. J. Warburton, Nat. Mater. 12, 483 (2013).

[4] P. Lodahl, S. Mahmoodian, and S. Stobbe, arXiv:1312.1079 [Rev. Mod. Phys. (to be published)].

[5] P. Michler, A. Kiraz, C. Becher, W. V. Schoefeld, P. M. Petroff, L. Zhang, E. Hu, and A. Imamoğlu, Science 290, 2282 (2000).

[6] C. Santori, M. Pelton, G. Solomon, Y. Dale, and Y. Yamamoto, Phys. Rev. Lett. 86, 1502 (2001).

[7] Q. Wang, S. Stobbe, and P. Lodahl, Phys. Rev. Lett. 107, 167404 (2011).

[8] D. Englund, D. Fattal, E. Waks, G. Solomon, B. Zhang, T. Nakaoka, Y. Arakawa, Y. Yamamoto, and J. Vučković, Phys. Rev. Lett. 95, 013904 (2005).

[9] H. Kamada, H. Gotoh, J. Temmyo, T. Takagahara, and H. Ando, Phys. Rev. Lett. 87, 246401 (2001); A. J. Ramsay, A. M. Fox, M. S. Skolnick, A. V. Gopal, E. M. Gauger, B. W. Lovett, and A. Nazir, Phys. Rev. Lett. 104, 017402 (2010).

[10] H. Jayakumar, A. Predojević, T. Huber, T. Kauten, G. S. Solomon, and G. Weihs, Phys. Rev. Lett. 110, 135505 (2013).

[11] X. Xu, B. Sun, P. R. Berman, D. G. Steel, A. S. Bracker, D. Gammon, and L. J. Sham, Science 317, 929 (2007).

[12] X. Xu, B. Sun, E. D. Kim, K. Smirl, P. R. Berman, D. G. Steel, A. S. Bracker, D. Gammon, and L. J. Sham, Phys. Rev. Lett. 101, 227401 (2008).

[13] A. Muller, E. B. Flagg, P. Bianucci, X. Y. Wang, D. G. Deppe, W. Ma, J. Zhang, G. J. Salamo, M. Xiao, and C. K. Shih, Phys. Rev. Lett. 99, 187402 (2007).

[14] A. N. Vamivakas, Y. Zhao, C.-Y. Lu, and M. Ataüre, Nat. Phys. 5, 198 (2009).

[15] E. B. Flagg, A. Muller, J. W. Robertson, S. Founta, D. G. Deppe, M. Xiao, W. Ma, G. J. Salamo, and C. K. Shih, Nat. Phys. 5, 203 (2009).

[16] E. B. Flagg, J. W. Robertson, S. Founta, W. Ma, M. Xiao, G. J. Salamo, and C. K. Shih, Phys. Rev. Lett. 102, 097402 (2009).

[17] S. M. Ulrich, S. Ates, S. Reitzenstein, A. Löffler, A. Forchel, and P. Michler, Phys. Rev. Lett. 106, 247402 (2011).
[18] A. N. Vamivakas, C.-Y. Lu, C. Matthiesen, Y. Zhao, S. Fält, A. Badolato, and M. Atatüre, Nature (London) 467, 297 (2010).

[19] S. Ates, S. M. Ulrich, S. Reitzenstein, A. Löffler, A. Forchel, and P. Michler, Phys. Rev. Lett. 103, 167402 (2009).

[20] Y.-M. He, Y. He, Y.-J. Wei, D. Wu, M. Atatüre, C. Schneider, S. Höfling, M. Kamp, C.-Y. Lu, and J.-W. Pan, Nat. Nanotechnol. 8, 213 (2013).

[21] C. Matthiesen, M. Geller, C.H. H. Schulte, C. L. Gall, J. Hansom, Z. Li, M. Hugues, E. Clarke, and M. Atatüre, Nat. Commun. 4, 1600 (2013).

[22] T. Rudolph, H. S. Freedhoff, and Z. Ficek, J. Opt. Soc. Am. B 15, 2325 (1998).

[23] Y. Ota, S. Iwamoto, N. Kumagai, and Y. Arakawa, Phys. Rev. Lett. 107, 233602 (2011).

[24] S.-Y. Zhu and M. O. Scully, Phys. Rev. Lett. 76, 388 (1996).

[25] H. Lee, P. Polynkin, M. O. Scully, and S.-Y. Zhu, Phys. Rev. A 55, 4454 (1997).

[26] Z. Ficek and T. Rudolph, Phys. Rev. A 60, R4245 (1999).

[27] Z. Ficek and H. S. Freedhoff, Phys. Rev. A 53, 4275 (1996).

[28] The Rabi frequency here is defined as $2 \Omega$, which follows the definition in Refs. [26,27].

[29] H.-R. Xia, C.-Y. Ye, and S.-Y. Zhu, Phys. Rev. Lett. 77, 1032 (1996).

[30] C. C. Yu, J. R. Bochinski, T. M. V. Kordich, T. W. Mossberg, and Z. Ficek, Phys. Rev. A 56, R4381(R) (1997).

[31] L. Li, X. Wang, J. Yang, G. Lazarov, J. Qi, and A. M. Lyyra, Phys. Rev. Lett. 84, 4016 (2000).

[32] P. R. Berman, Phys. Rev. A 58, 4886 (1998).

[33] J. Wang, H. M. Wiseman, and Z. Ficek, Phys. Rev. A 62, 013818 (2000).

[34] M. Peiris, K. Konthasinghe, Y. Yu, Z. C. Niu, and A. Muller, Phys. Rev. B 89, 155305 (2014).

[35] See Supplemental Material at http://link.aps.org/ supplemental/10.1103/PhysRevLett.114.097402, for details of the experimental setup, sample information and calculations.

[36] J. R. Bochinski, C. C. Yu, T. Loftus, and T. W. Mossberg, Phys. Rev. A 63, 051402(R) (2001).

[37] K. Bergmann, H. Theuer, and B. W. Shore, Rev. Mod. Phys. 70, 1003 (1998); X. Xu, B. Sun, P. R. Berman, D. G. Steel, A. S. Bracker, D. Gammon, and L. J. Sham, Nat. Phys. 4, 692 (2008); D. Brunner, B. D. Gerardot, P. A. Dalgarno, G. Wüst, K. Karrai, N. G. Stoltz, P. M. Petroff, and R. J. Warburton, Science 325, 70 (2009).

[38] M. Mücke, E. Figueroa, J. Bochmann, C. Hahn, K. Murr, S. Ritter, C. J. Villas-Boas, and G. Rempe, Nature (London) 465, 755 (2010).

[39] H. S. Freedhoff and Z. Ficek, Phys. Rev. A 55, 1234 (1997).

[40] P. Zhou and S. Swain, Phys. Rev. Lett. 77, 3995 (1996); Z. Ficek and S. Swain, Phys. Rev. A 69, 023401 (2004); F.-L. Li and S.-Y. Zhu, Phys. Rev. A 59, 2330 (1999); E. Paspalakis and P. L. Knight, Phys. Rev. Lett. 81, 293 (1998); M. Kiffner, J. Evers, and C. H. Keitel, Phys. Rev. A 73, 063814 (2006).

[41] P. Zhou and S. Swain, Phys. Rev. Lett. 78, 832 (1997); S. E. Harris, Phys. Rev. Lett. 62, 1033 (1989).

[42] Z.-H. Tang, G.-X. Li, and Z. Ficek, Phys. Rev. A 82, 063837 (2010); J. Cai, G. G. Guerreschi, and H. J. Briegel, Phys. Rev. Lett. 104, 220502 (2010). 